\title{
Desde la corteza auditiva a la cóclea: \\ Progresos en el sistema eferente auditivo
}

\section{From auditory cortex to cochlea: Progress in the auditory efferent system}

\author{
Gonzalo Terreros H¹, Bárbara Wipe U', Alex León I', y Paul H. Délano R ${ }^{1,2}$.
}

\begin{abstract}
RESUMEN
El sistema eferente auditivo está constituido por el sistema olivococlear y por vías descendentes que provienen de la corteza auditiva y se dirigen a la cóclea. El sistema olivococlear se divide en una porción medial y una lateral, con neuronas que inervan a las células ciliadas externas y a fibras del nervio auditivo respectivamente. El principal neurotransmisor de las sinapsis olivococleares es acetilcolina, y tanto las células ciliadas externas como las fibras del nervio auditivo poseen receptores para esta molécula. El sistema eferente córtico-coclear se origina en la capa Vy VI de la corteza auditiva y proyecta a los colículos inferiores y complejo olivar superior, donde a través del sistema olivococlear se conecta con el órgano receptor auditivo. En este artículo se revisan importantes hallazgos obtenidos en los últimos años que involucran (i) nuevos neurotransmisores y receptores del sistema eferente auditivo; (ii) vías descendentes de la corteza auditiva y su rol fisiológico sobre las respuestas cocleares y (iii) rol del sistema eferente auditivo en patologías audiológicas y neuropsiquiátricas.
\end{abstract}

Palabras clave: Olivococlear, sistema eferente, vías descendentes, corteza auditiva, cóclea, neurotransmisores.

\begin{abstract}
The auditory efferent system is composed by the olivocochlear fibers and descending projections that originate in the auditory cortex and end in the cochlea. The olivocochlear system is divided into a medial and lateral division, with fibers directed to the outer hair cells and to the auditory nerve fibers respectively. It is known that acetylcholine is the main neurotransmitter of the olivocochlear synapses and that outer hair cells and auditory nerve fibers have receptors to this molecule. The cortico-cochlear efferent system originates in layers $V$ and $\mathrm{VI}$ of the auditory cortex. These descending projections are directed to the inferior colliculus and superior olivary complex, a site in which the olivocochlear fibers emerge and connect the brain with the cochlear receptor. In this article recent discoveries obtained in the last years are reviewed: (i) new neurotransmitters and receptors of the olivocochlear system; (ii) anatomy and physiology of descending pathways from the auditory cortex to the cochlea and, (iii) clinical role of auditory efferents in audiological and neuropsychiatric pathologies.

Key words: Olivocochlear, auditory efferent, descending projections, auditory cortex, cochlea, neurotransmitters.
\end{abstract}

\footnotetext{
${ }^{1}$ Programa de Fisiología y Biofísica, ICBM, Facultad de Medicina, Universidad de Chile.

${ }^{2}$ Servicio de Otorrinolaringología, Hospital Clínico de la Universidad de Chile.
} 


\section{INTRODUCCIÓN}

La existencia de fibras eferentes auditivas, originadas en el tronco encefálico y dirigidas hacia la cóclea, fueron inicialmente descritas por Rasmussen en 1946 y 1960 siendo éstas denominadas como fibras olivococleares cruzadas y no cruzadas respectivamente $^{1,2}$. Posteriormente, entre 1975 y 1979, Warr y Guinan describieron el origen neuroanatómico de estas vías y clasificaron al sistema olivococlear en medial y lateral ${ }^{3}$. Las fibras olivococleares mediales inervan a las células ciliadas externas y su principal neurotransmisor es acetilcolina (ACh), mientras que las fibras laterales inervan a las fibras aferentes del nervio auditivo y liberan diversos neurotransmisores como ACh, dopamina y opioides ${ }^{4,5}$ (Figura 1).

Sin embargo, además del sistema olivococlear, se sabe que existen vías neurales descendentes que conectan la corteza auditiva con el sistema olivococlear, constituyendo el sistema eferente auditivo córtico-coclear. Este incluye dos circuitos, el primero se dirige al cuerpo geniculado medial del tálamo y el segundo a los colículos inferiores, complejo olivar superior y desde este núcleo a la cóclea ${ }^{6,7}$.

Por otra parte, si bien el rol del sistema eferente auditivo en la audición es aún controversial, se postulan varias hipótesis como: (i) protección frente a sonidos de alta intensidad8; (ii) efecto antienmascarador de estímulos auditivos en presencia de ruido de fondo $0^{9}$; (iii) atención selectiva ${ }^{10,11} \mathrm{y}$ (iv) modulación de la sensibilidad coclear durante el ciclo de sueño y vigilia ${ }^{12}$.

En el presente artículo se realiza una revisión enfocada en tres temas en los que se han realizado avances importantes en el entendimiento del sistema eferente auditivo en los últimos años: (i) Neurotransmisores de las sinapsis olivococleares; (ii) Fisiología del sistema cortico-coclear y (iii) Rol en clínica del sistema eferente auditivo. Para revisiones más generales del sistema eferente auditivo, el lector puede consultar artículos publicados en esta revista 0 en libros especializados ${ }^{4,13,14}$.

\section{NEUROTRANSMISORES DE LAS SINAPSIS OLIVOCOCLEARES}

El sistema olivococlear está compuesto de fibras originadas en el complejo olivar superior y que de acuerdo estudios realizados con trazadores neuronales retrógrados inyectados en la cóclea, se divide en medial (OCM) y lateral (OCL) ${ }^{3}$. El sistema OCM posee fibras mielinizadas que cruzan a nivel del piso del cuarto ventrículo llegando mayoritariamente a la cóclea contralateral. El sistema OCL, está compuesto por fibras amielínicas que proyectan a la cóclea ipsilateral2,4. A nivel coclear, las sinapsis de las fibras del sistema OCM sinaptan a las células ciliadas externas (CCE) ${ }^{15}$, mientras que los terminales de los axones del sistema OCL hacen sinapsis con las dendritas del nervio auditivo próximas a las células ciliadas internas $(\mathrm{CCl})^{16}$.

\section{NEUROTRANSMISORES DEL SISTEMA OLIVOCOCLEAR LATERAL}

El uso de la tinción de colinesterasa ${ }^{15}$ y posteriormente de tinción de colina acetil-transferasa ${ }^{17}$, reveló que la modulación y transmisión sináptica entre las fibras OCL y las fibras aferentes primarias del nervio auditivo son dependientes principalmente de ACh, así como de otros neurotransmisores y neuromoduladores tales como: ácido gama aminobutírico (GABA), dopamina, dinorfinas, encefalinas, el péptido relacionado al gen de calcitonina (PRGC) serotonina y glicina ${ }^{16,18,19}$ (Figura 2).

\section{Acetilcolina (OCL)}

ACh es el principal neurotransmisor liberado por los terminales olivococleares, y tanto el sistema OCL como el OCM poseen receptores postsinápticos de tipo colinérgicos ${ }^{18,20}$. La liberación de ACh en el sistema OCL fue descrita en 1975 utilizando marcadores axonales retrógrados en gatos ${ }^{15}$. Posteriormente, la presencia de ACh en el haz OCL fue descrita en otros modelos animales como el conejillo de indias (Cavia porcellus) ${ }^{21}$ y confirmada a través de diferentes técnicas histoquímicas utilizando microscopía electrónica y anticuerpos monoclonales contra colina-acetiltranferasa ${ }^{22,23}$. La ACh actúa sobre receptores nicotínicos del tipo $\alpha 7$ (Figura 2) en el sistema OCL, siendo estos expresados desde la etapa embrionaria hasta la vida adulta ${ }^{24}$.

\section{GABA (OCL)}

GABA es el principal neurotransmisor inhibitorio en el sistema nervioso central. Utilizando ensayos 


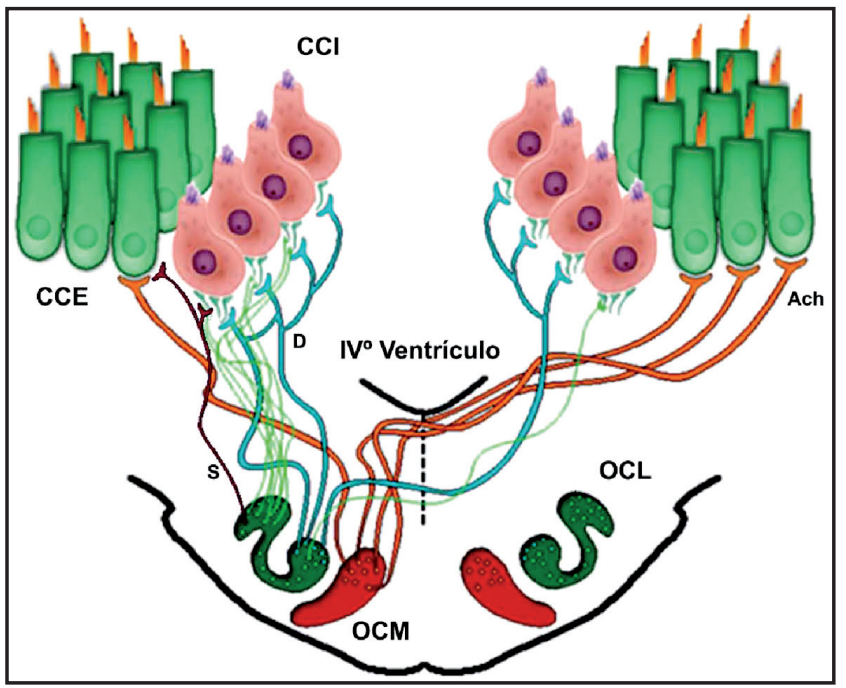

Figura 1.Sistema olivococlear. La figura muestra el sistema olivococlear medial (OCM) y lateral (OCL). En el complejo olivar se encuentran tres tipos de neuronas olivococleares: (i) Neuronas intrínsecas del OCL (fibras en verde); (ii) las neuronas en caparazón (fibras celestes) y (iii) las neuronas del sistema OCM (fibras gruesas y en naranjo). El OCL está compuesto por un haz de fibras amielínicas que nacen en el complejo olivar superior y proyectan en forma ipsilateral inervando a fibras del nervio auditivo cerca de las sinapsis con las CCI. Diversos neurotransmisores han sido descritos en la conectividad OCLCCI (ver texto), mostrándose acá solo Serotonina (S) y Dopamina (D) como mediadores de esta sinapsis. El haz OCM corresponde a un conjunto de fibras mielinizadas que proyectan mayoritariamente a la cóclea contralateral cruzando por el piso del IV ventrículo inervando a las CCE (CCI: Célula ciliada interna; CCE: Célula ciliada externa; Ach: Acetilcolina).

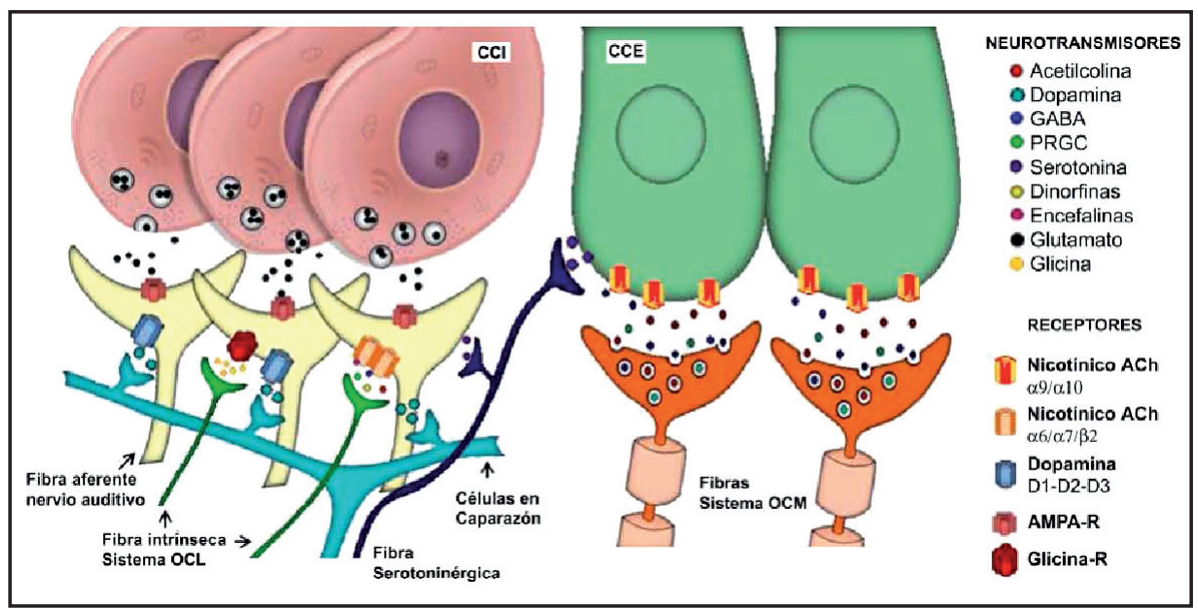

Figura 2. Neurotransmisores involucrados en la conectividad del sistema olivococlear con células ciliadas internas y externas de la cóclea.

El sistema OCL utiliza diversos neurotransmisores en la conectividad con las CCl. Las fibras intrínsecas del OCL (en verde) sinaptan directamente en el nervio auditivo utilizando como neurotransmisores ACh, Dinorfinas, PRCG, GABA y Glicina. Por otro lado han sido descritas fibras serotoninérgicas (en azul) que hacen sinapsis con la fibra aferente auditiva y con células ciliadas externas adyacentes al túnel de Corti. Las neuronas en caparazón (en celeste) utilizan a dopamina como su principal neurotransmisor y actúan sobre receptores dopaminérgicos (D1D3). El principal neurotransmisor liberado por las fibras del sistema OCM y que sinapta directamente sobre las CCE es ACh el cual actúa sobre un receptor con características particulares conteniendo subunidades de tipo $\alpha 9 / \alpha 10$. Otros neurotransmisores descritos en esta sinapsis son dopamina, GABA, encefalinas y PRCG. (CCl: célula ciliada interna; CCE: célula ciliada externa, ACh: acetilcolina; PRCG: péptido relacionado al gen de calcitonina). 
de inmuno-reactividad, se descubrió que los terminales sinápticos gabaérgicos se localizan en la base de las CCE y también formando parte del sistema OCL ${ }^{18}$. La microinyección de GABA en la vecindad de las $\mathrm{CCl}$ disminuye la respuesta producida por aminoácidos excitatorios, pero tiene poco efecto en la actividad espontánea de estas fibras ${ }^{16,18}$. GABA en el oído interno tendría funciones inhibitorias, permitiendo regular la actividad del nervio auditivo y la transmisión de información auditiva al cerebro.

\section{Dopamina (OCL)}

La presencia de neuronas de tipo dopaminérgicas en la cóclea, formando parte del sistema OCL, fue descrita inicialmente a través de evidencias obtenidas por cromatografía líquida ${ }^{25}$ y constatadas por Eybalin a través de sus estudios de microscopía ${ }^{17}$. La dopamina podría tener la función de reducir el daño neural frente a sonidos de alta intensidad, de acuerdo con lo concluido a partir de estudios en los que se utilizaba Piribedil, un agonista de receptores D226,27. Estos resultados fueron ratificados por los experimentos realizados por el grupo del Dr. Puel, en los que la aplicación de dopamina intracoclear generó un aumento del umbral excitatorio y una reducción de la tasa de descarga de fibras del nervio auditivo, resultando en una disminución del potencial de acción compuesto del nervio auditivo (PAC). El mecanismo propuesto indicaría que la dopamina actuaría a nivel posináptico, regulando la tasa de descarga de las neuronas aferentes auditivas ${ }^{28}$.

\section{Opioides y PRGC (OCL)}

Las encefalinas fueron descritas en el cerebro desde mediados de los años $70^{18}$, pero caracterizadas en el sistema eferente auditivo una década después ${ }^{29}$. Se postula que las encefalinas tienen funciones inhibitorias dentro del sistema auditivo de mamíferos, rol funcional que ha sido constatado de manera indirecta a través de la verificación del aumento de su concentración en ambientes ruidosos y disminución en ambientes silenciosos ${ }^{16}$.

Las dinorfinas son otros péptidos pertenecientes a la familia de las endorfinas y cuyo precursor es prodinorfina ${ }^{18}$. La presencia de dinorfina $B$ en la cóclea de mamíferos fue inicialmente descrita por
Hoffman y cols $(1985)^{30}$. Se cree que su función es modular la actividad del nervio auditivo $0^{30}$, y específicamente se le ha asignado una función excitatoria, potenciando los efectos del glutamato en la actividad del nervio auditivo ${ }^{23}$.

A través de inmunocitoquímica se obtuvo evidencia de la presencia de PRGC en un haz de neuronas que ingresan a la cóclea y que hacen sinapsis principalmente con las $\mathrm{CCl}^{31}$, aunque también con las CCE ${ }^{18}$, Matsunaga y cols (1986) ${ }^{32}$ asignan a PRGC una función inhibitoria sobre la actividad del nervio auditivo ya que produce una reducción dosis dependiente de la amplitud del $\mathrm{PAC}^{16}$. Estos resultados, se contraponen a los resultados reportados por Adams y cols en 198733,34 quienes asignan un papel excitatorio al PRGC. Es probable que el PRCG cumpla un papel modulador de las descargas del nervio auditivo, siendo aún controversial su efecto predominante.

\section{Serotonina (OCL)}

Los primeros trabajos que exploraron la presencia de fibras serotoninérgicas inervando la cóclea provienen de estudios con primates (Otolemurgarnetti) en los cuales, utilizando doble tinción histológica y microscopía óptica se encontró inervación en Ios sistemas OCL y $\mathrm{OCM}^{35}$. Años después utilizando gatos $^{36}$ y ratas $^{37}$ se encontraron fibras serotoninérgicas inervando principalmente las $\mathrm{CCl}$ y, aunque en menor medida, también, las CCE ${ }^{38}$. El origen de esta inervación fue inicialmente un enigma descartándose tempranamente como origen de estas proyecciones el ganglio cervical superior ${ }^{37}$ y posteriormente estableciéndose como origen más probable de estas fibras neuronas del tronco encefálico ubicadas alrededor del complejo olivar superior y del núcleo periolivar ${ }^{39}$. La función específica de esta inervación serotoninérgica no es del todo clara, sin embargo, en experimentos en los que se utilizan bloqueadores de fibras serotoninérgicas se ha concluido que estas fibras estarían involucradas en el control de ganancia de los receptores auditivos, aunque también se ha relacionado a cambios del estado de alerta, atención y control del ciclo sueño-vigilia ${ }^{39}$.

\section{Glicina (OCL)}

Si bien los principales neurotransmisores involucrados en las sinapsis inhibitorias OCL son ACh, 
dopamina y GABA, el año 2008 fue descrito como un importante componente de las sinapsis eferentes del sistema nervioso auditivo a la glicina ${ }^{40}$. Dos tipos de receptores de glicina fueron descritos a través de las técnicas de PCR, hibridación in-situ e inmunohistoquímica en el órgano de Corti y neuronas del ganglio espiral en rata adulta ${ }^{40}$. Posteriormente, se describieron otros receptores de glicina, predominando en los estadios P0-P21 del desarrollo del órgano de Corti del murino ${ }^{41}$. Estos antecedentes permiten a los autores plantear un paralelo entre la translocación de los receptores de glicina y el cambio de la inervación eferente coclear desde las CCI a las CCE que ocurre durante el desarrollo temprano del oído interno ${ }^{41}$.

\section{NEUROTRANSMISORES DEL SISTEMA OLIVOCOCLEAR MEDIAL}

\section{Acetilcolina (OCM)}

El haz OCM está compuesto por axones mielinizados provenientes de neuronas de gran tamaño ubicadas en el complejo olivar medial y que sinaptan a las CCE ${ }^{4}$. De esta manera las fibras eferentes OCM podrían modular la ganancia del amplificador coclear que se localiza en las CCE ${ }^{42}$, mientras que las fibras aferentes que inervan a las CCE sólo constituyen entre 5\%-10\% del total de fibras aferentes del nervio auditi$\mathrm{v}^{43,44}$. El principal neurotransmisor de la sinapsis eferente sobre las CCE es ACh, el cual, se ha propuesto que actúa sobre receptores nicotínicos, pero también muscarínicos en las CCE ${ }^{45}$. Sin embargo, los datos farmacológicos y electrofisiológicos han sugerido un rol central de un receptor atípico de tipo nicotínico localizado en las sinapsis entre las fibras eferentes y las $\mathrm{CCE}^{46}$. Se ha propuesto que el receptor nicotínico que media las sinapsis colinérgicas entre OCM y CCE está compuesto de una estructura pentamérica (Figura 2) conformado por subunidades $\alpha 9$ y $\alpha 10^{46}$. La evidencia indica que la activación de este receptor permite un incremento de la concentración intracelular de $\mathrm{Ca}^{2+}$ en las CCE y así la apertura de canales de $\mathrm{K}^{+}(\mathrm{SK} 2)^{47}$ generando hiperpolarización de las células ciliadas y reducción de la electromotilidad ${ }^{48}$.

\section{GABA (OCM)}

A través de estudios histológicos utilizando anticuerpos contra GABA se ha encontrado inmuno- reactividad en cuerpos celulares en el complejo olivar superior y en terminales localizados bajos las células ciliadas (Figura 2) ${ }^{17}$. Si bien el rol de las neuronas gabaérgicas en el eferente auditivo no es del todo entendido ${ }^{49}$, se ha sugerido que podría tener funciones inhibitorias en las CCE. A partir de los resultados obtenidos en estudios de canales iónicos en un modelo de CCE aislada, se ha demostrado que el GABA genera hiperpolarización celular que depende la concentración utilizada. Este efecto sería potenciado por agonistas como benzodiacepinas e inhibido por bloqueadores de receptores GABA como Picrotoxina ${ }^{50}$. Estos resultados sugieren que el GABA podría estar actuando sobre receptores de tipo GABA A generando hiperpolarización de las CCE.

\section{PRGC (OCM)}

Basado en estudios neuroanatómicos se ha propuesto al PRCG como un neuromodulador de las sinapsis eferentes del sistema auditivo ${ }^{18}$. Han sido identificados terminales PRCG en las CCI y en menor medida también en la sinapsis OCMCCE $^{31,51,52}$, lo cual ha sido confirmado a través de inmunocitoquímica e hibridación in situ ${ }^{17}$. La función que ejerce PRCG en la sinapsis OCM-CCE resulta desconocida.

\section{FISIOLOGÍA DEL SISTEMA CÓRTICO-COCLEAR}

El sistema eferente auditivo no sólo comprende al sistema olivococlear, sino que además posee vías descendentes que se originan en la corteza auditiva y se dirigen al colículo inferior, al complejo olivar superior y al tálamo auditivo (Figura $3)^{53,54}$. Estas vías descendentes tienen su origen en neuronas piramidales ubicadas en la capa $V y$ VI de la corteza auditiva primaria ${ }^{14,54,55}$. Desde aquí proyectan dos vías principales, la primera hacia el cuerpo geniculado medial del tálamo, y la segunda hacia otros núcleos subcorticales, principalmente ipsilaterales a la corteza, como colículos inferiores, complejo olivar superior y núcleos cocleares ${ }^{56,57}$.

Electrofisiológicamente, se ha descrito que existen dos poblaciones de neuronas piramidales en la capa V de la corteza auditiva ${ }^{58}$. Estas neuronas poseen proyecciones glutamatérgicas (excitatorias) 
al colículo inferior y desde el colículo inferior al complejo olivar superior ${ }^{59,60}$. La funcionalidad del sistema córtico-olivococlear ha sido evaluada en experimentos en murciélagos, chinchillas y también en seres humanos ${ }^{61,62,63}$. Xiao y Suga publicaron un trabajo seminal en el año 2001, donde demostraron que la microestimulación eléctrica de la corteza auditiva del murciélago mostachudo (Pteronotusparnellii) modifica la amplitud del potencial microfónico coclear en la frecuencia de ecolocalización de este murciélago $(61 \mathrm{kHz})$. Por otra parte León y cols (2012) ${ }^{62}$ demostraron a través de experimentos de inactivación de la corteza auditiva en chinchillas (Chinchilla laniger) que el efecto córtico-coclear encontrado por Xiao y Suga también se puede obtener en mamíferos no ecolocalizadores, en frecuencias de 1 a $8 \mathrm{kHz}$, y además de que existe un tono cortical de actividad eferente que regula la sensibilidad coclear. En las Figuras 4 y 5 se muestran ejemplos de cómo la inactivación con microinyecciones de lidocaína en la corteza auditiva de chinchillas modifica la amplitud del potencial microfónico coclear y del PAC del nervio auditivo, tanto en el oído ipsi como contralateral a la corteza auditiva inactivada.

En seres humanos se han realizado experimentos en pacientes con epilepsia del lóbulo temporal refractaria a tratamiento farmacológico que requieren una neurocirugía para resección de su foco epileptogéni$\mathrm{CO}^{63}$. En estos pacientes se realizó microestimulación de la corteza auditiva y medición simultánea de emisiones otoacústicas durante el evento quirúrgico. En ellos se observó una reducción de amplitud de las emisiones otoacústicas al microestimular la corteza auditiva, y no cuando se microestimulan otras áreas de la corteza cerebral. De esta manera, los hallazgos obtenidos en modelos animales y en pacientes epilépticos muestran que la corteza auditiva regula la sensibilidad coclear en mamíferos a través del sistema eferente auditivo. En la Figura 6 se muestra un modelo del funcionamiento del sistema eferente auditivo, desde la corteza auditiva hasta la cóclea, donde se plantea la existencia de vías paralelas desde la corteza auditiva hasta el OCM y OCL.

\section{ROL CLÍNICO DEL SISTEMA EFERENTE AUDITIVO}

Se ha descrito una serie de patologías auditivas, neurológicas y psiquiátricas cuya etiología o fisiopato- logía podría manifestarse con una disfunción eferente: (i) tinnitus, (ii) presbiacusia,(iii) trauma acústico (protección), (iv) miastenia gravis, (v) déficit atencional, (vi) mutismo selectivo y (vii) otras. A continuación se revisa la evidencia sobre el rol del sistema eferente auditivo en cada una de estas patologías.

\section{(i) Sistema eferente auditivo y tinnitus}

Se ha propuesto una alteración de la función eferente auditiva en el grupo de pacientes con tinnitus que presentan audición y curvas impedanciométricas normales ${ }^{64}$. Sin embargo estos hallazgos son controversiales ya que otros autores no han encontrado diferencias entre sujetos controles y aquellos con tinnitus ${ }^{65-67}$.

Si bien no es claro el rol del sistema eferente auditivo en la generación del tinnitus, dado su función predominantemente inhibitoria sobre las respuestas cocleares, actualmente se han generado fármacos en que se potencia la función inhibitoria del sistema eferente para su posible uso en pacientes con tinnitus $^{46}$. Específicamente se está realizando un ensayo clínico en pacientes con tinnitus con neramexano, una molécula fabricada por el Laboratorio $\mathrm{Merz}^{\circledR}$ de Alemania, que es un bloqueador de receptores NMDA de glutamato y del receptor nicotínico $\alpha$ 9/ $\alpha-10$ de las CCE cocleares. Hasta el momento, el fármaco ha mostrado un efecto supresor del tinnitus superior al de placebo $0^{68}$. Dada la variedad de neurotransmisores presentes en los sistemas OCM y $\mathrm{OCL}$, es probable que un futuro cercano emerjan nuevos tratamientos farmacológicos que intenten manipular la función eferente auditiva.

\section{(ii) Sistema eferente auditivo y presbiacusia}

Estructuralmente, la presbiacusia se caracteriza por la pérdida progresiva de neuronas ganglionares y de células ciliadas cocleares en relación al envejecimiento ${ }^{69}$. Además, en la cepa de ratón C57BL/6J, que es un modelo de presbiacusia, se ha demostrado una disminución del número de sinapsis entre las $\mathrm{CCl}$ y las neuronas ganglionares ${ }^{70}$.

Estudios recientes realizados en ratones C57BL/6J muestran una reducción del número de sinapsis entre fibras OCM y CCE con el envejecimiento del animal ${ }^{71}$. En concordancia con estos resultados anatómicos, funcionalmente se ha observado una disminución 
Figura 3. Sistema eferente córtico-coclear. El sistema eferente auditivo se origina principalmente en las capas V y VI de la corteza auditiva primaria permitiendo regular la función coclear. Las vías descendentes proyectan a través de dos circuitos, el primero sinapta con neuronas del tálamo auditivo (cuerpo geniculado medial) y la segunda se dirige a otros núcleos subcorticales, tales como el colículo inferior, el núcleo coclear y el complejo olivar superior. En la parte medial de la oliva superior emergen fibras neuronales que proyectan de manera contralateral a la cóclea generando las fibras $\mathrm{OCM}(\mathrm{O} \mathrm{MOC})$, mientras que las fibras $O C L$ (0 LOC) inervan principalmente a las fibras ipsilaterales del nervio auditivo. La información auditiva aferente va desde la cóclea enviando esta información a los núcleos cocleares a través del nervio auditivo (flechas en negro).

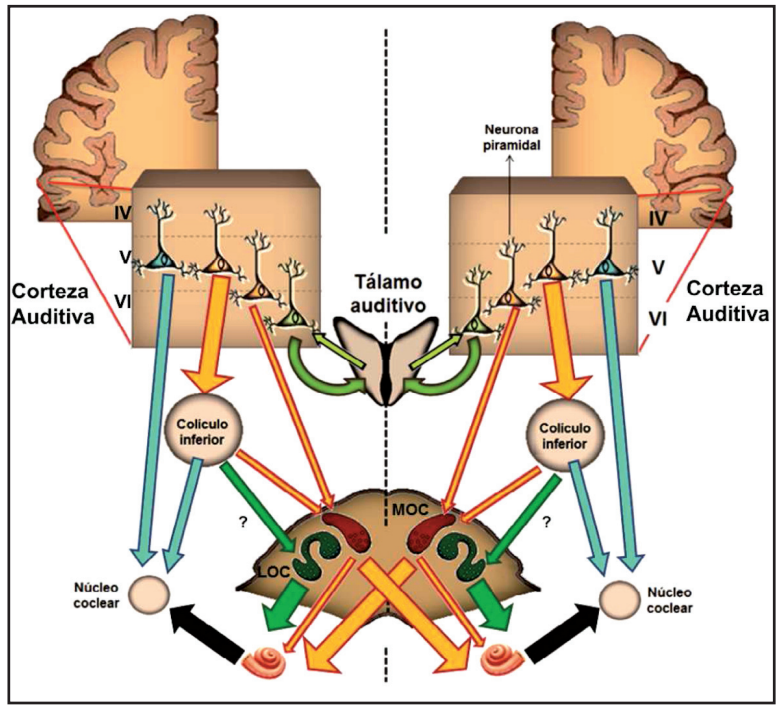

Figura 4. Efecto de la inactivación de la corteza auditiva con microinyecciones de lidocaína en la amplitud de los potenciales cocleares. Los potenciales cocleares, potencial de acción compuesto del nervio auditivo (CAP) y potencial microfónico coclear (CM) fueron registrados antes (trazos negros) y después (trazos rojos) de la inactivación de la corteza auditiva con microinyecciones de lidocaína. Los ejemplos que se muestran en el panel A y B fueron obtenidas en diferentes experimentos. A. Se muestra un incremento significativo de amplitud del CAP junto a una reducción del CM después de la microinyección de lidocaína. Los estímulos auditivos fueron presentados a $4 \mathrm{kHz}$ y a diferentes niveles de presión sonora. B. Se muestra una reducción del CAP y de CM después de la microinyección de lidocaína. En este ejemplo los tonos fueron presentados a $2 \mathrm{kHz}$ y a diferentes niveles de presión sonora. (Figura modificada de León y cols., PLoS One, 2012; 7(4): e36203. doi: 10.1371/journal.pone.0036203).

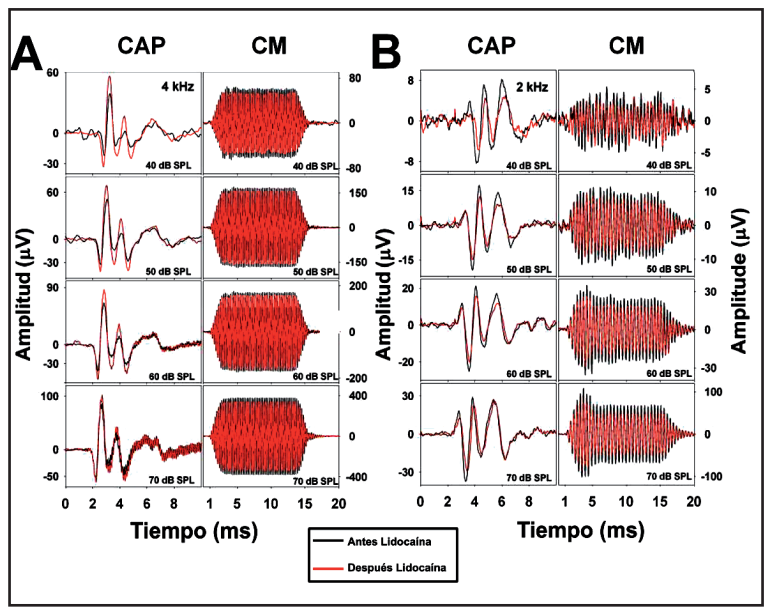

Figura 5. Curso temporal de los cambios de amplitud en los potenciales corticales y cocleares antes y después de la inactivación cortical con lidocaína. Los cambios de amplitud son representados como cambios en decibeles respecto a la amplitud de la línea base antes de la microinyección de lidocaína. La figura muestra los registros obtenidos del potencial CM (cuadrados azules), el CAP (círculos rojos) y los cambios de amplitud del potencial evocado cortical (triángulo negro). Se presentó un tono de $6 \mathrm{kHz}$ a diferentes niveles de presiones sonoras, incrementando en pasos de $10 \mathrm{~dB}$ entre 30 a $90 \mathrm{~dB}$ SPL, lo que se representa en el tamaño de los símbolos. Se observó un aumento significativo del CAP y del potencial CM después de la microinyección de 3 I de lidocaína en la corteza auditiva contralateral a la cóclea estudiada. (Figura modificada de León y cols., PLoS One, 2012;7(4): e36203. doi: 10.1371/journal.pone.0036203).

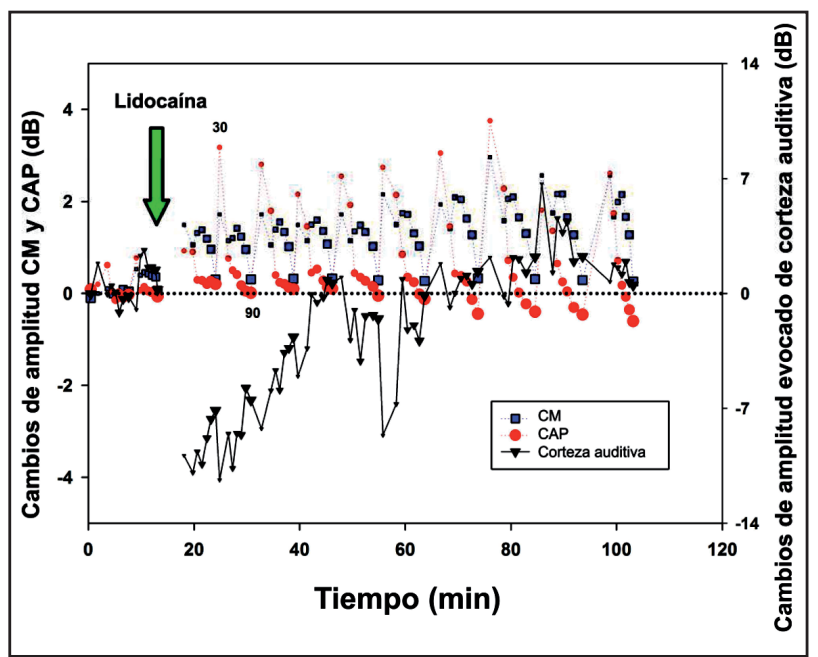




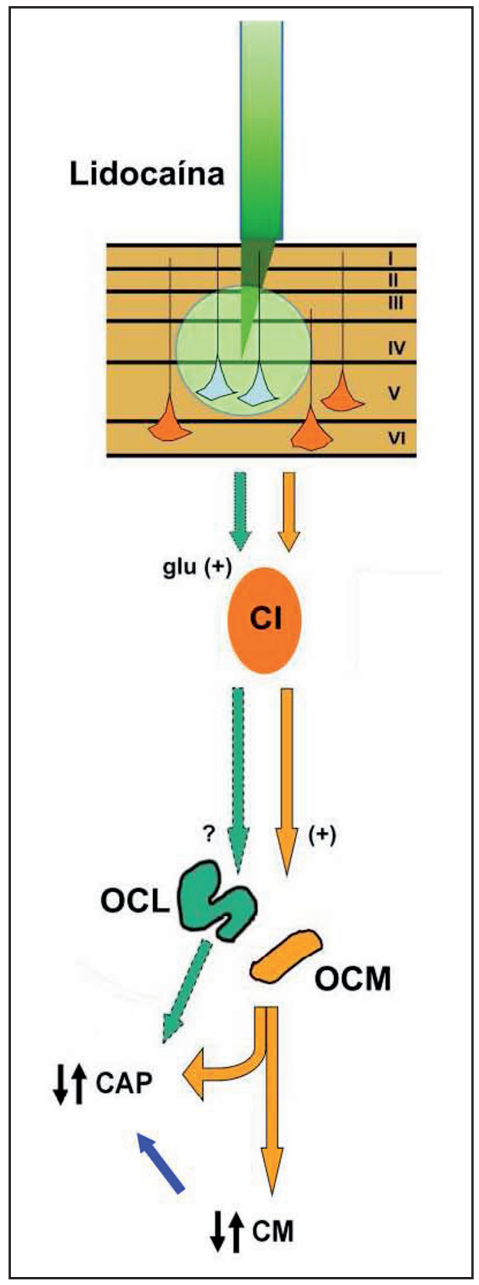

Figura 6. Modelo de los efectos de la microinyección de lidocaína en la corteza auditiva sobre la conectividad córtico-coclear. Representación esquemática del modelo de trabajo utilizado para el estudio de la modulación corticofugal en la repuesta del nervio auditivo y de la cóclea. Basado en estudios previos León y cols (2012). Se propone la presencia de un tono basal excitatorio mediado por dos grupos de neuronas piramidales glutamatérgicas ubicadas en la capa V y VI de la corteza auditiva y que proyectan en forma paralela hacia el (colículo inferior) Cl y el sistema OCM. La microinyección de lidocaína en la corteza auditiva (área sombreada verde) en el modelo propuesto, genera una perdida de actividad de las vías excitatorias descendientes produciendo cambios en el potencial CM y en el CAP. La inactivación azarosa de diferentes subpoblaciones de neuronas piramidales, podrían en parte explicar, las diferencias interindividuos encontradas. Las flechas azules desde CM al CAP representan una hipótesis alternativa para explicar los cambios de amplitud del CAP. El signo de interrogación representa vías no descritas, pero que probablemente existen entre el colículo inferior y núcleo olivar superior lateral. (IVI: capas de la corteza auditiva; glu: glutamato; Cl: coliculo inferior; (+): actividad excitatoria) (Figura modificada de León y cols., PLOS One, 2012; 7(4): e36203. doi: 10.1371/journal.pone.0036203). de la magnitud del reflejo acústico olivococlear en ratones CBA de edad avanzada ${ }^{72}$. Incluso, se postula que esta reducción funcional del sistema eferente auditivo se produce antes que la disminución del número de CCE relacionada al envejecimiento ${ }^{73}$.

Recientemente se ha propuesto que el déficit de alfa-sinucleína, una proteína que se localiza en los terminales de los axones eferentes, sería una posible causa de presbiacusia en ratones C57BL/6 ${ }^{74}$. Si bien, aún no se sabe si estos hallazgos en modelos animales se producen también en seres humanos, si así fuese, determinar la función eferente en pacientes adultos normoyentes podría ayudar a predecir quiénes van a desarrollar presbiacusia y por lo tanto intentar impedir su desarrollo.

\section{(iii) Protección contra trauma acústico y sistema eferente auditivo}

Se ha reportado una disminución en la magnitud de la supresión contralateral de emisiones otoacústicas transientes en pacientes que presentan tinnitus secundario a un trauma acústico crónico ${ }^{75}$. Existe evidencia en estudios con modelos animales de que una disminución de la magnitud de la supresión olivococlear con ruido contralateral provoca mayor susceptibilidad al daño por sonidos de alta intensidad $^{8}$, de esta forma, el origen del tinnitus en pacientes expuestos a trauma acústico crónico podría explicarse por una disfunción eferente.

El desarrollo de un ratón genéticamente modificado ( $\alpha$-9-L9'T knock-in) que muestra una función eferente incrementada permitió entender mejor la función del sistema eferente auditiv ${ }^{76}$. Este ratón tiene mayor resistencia a la exposición a ruido de alta intensidad, que se explica por la inhibición de mayor magnitud que se produce al estimular al sistema eferente. En esta línea de investigación, el grupo de la Dra. Elgoyhen de la Universidad de Buenos Aires, Argentina está intentando desarrollar fármacos que potencien la función eferente, de manera de administrárselo a sujetos que se sabe serán expuestos a sonidos intensos (militares, metalúrgicos, obreros de la construcción, etc.) ${ }^{46}$.

\section{(iv) Miastenia gravis}

La miastenia gravis (MG) es una enfermedad autoinmune que se caracteriza por presentar autoan- 
ticuerpos dirigidos contra los receptores nicotínicos de ACh y que clínicamente se manifiesta con debilidad del músculo esquelético. Habitualmente los pacientes presentan ptosis palpebral, diplopía e incluso puede provocar parálisis de músculos respiratorios ${ }^{77}$. Para el tratamiento farmacológico se utilizan inhibidores de la acetilcolinesterasa, como piridostigmina ${ }^{77}$.

Debido a la patogenia de esta enfermedad y a la presencia de los receptores nicotínicos de ACh en las CCE, se ha buscado en forma dirigida una posible disfunción del sistema eferente auditiv0 ${ }^{77-79}$. En este sentido los estudios de Toth y cols (1998) ${ }^{80}$ demuestran que en pacientes que padecen MG, la función de los receptores de ACh presentes en las CCE está disminuida. En este contexto, Toth demuestra que los valores evaluados de las emisiones otoacústicas evocadas transientes (TOAE) fueron significativamente menores en pacientes con MG comparados con pacientes sanos (control). La utilización de un inhibidor reversible de la colinesterasa, Mestinon ${ }^{\circledR}$ (piridostigmina), generó un incremento en los valores de TOAE que no alcanzó los niveles observados en los sujetos controles ${ }^{80}$. Estos resultados indican que el sistema eferente auditivo resulta afectado en pacientes con MG. Un estudio llevado a cabo por el grupo de Hamed $(2006)^{77}$ en pacientes en un estado avanzado de MG, reclutados durante crisis miasténicas agudas o cuando sufrían de disfunción orofaríngea aguda, mostraron una significativa reducción de amplitud de los productos de distorsión de las emisiones otoacústicas (DPOAE) con una marcada reducción a la frecuencia de $5 \mathrm{kHz}$ comparado al grupo control ${ }^{77}$.

Durante este experimento la aplicación de un ruido contralateral (tonos, clics 0 ruido blanco) produjo una disminución significativa de las DPOAE y de TOAE en el grupo control y una reducción no significativa para la mayoría de las frecuencias evaluadas en pacientes que padecen MG y que fueron tratados durante dos meses ${ }^{77}$. Estos resultados pueden ser explicados por la disminución que genera la MG, de los receptores de ACh contenidos en las CCE en el oído interno, afectando la función del sistema eferente auditivo. Los autores concluyen que después de dos meses de tratamiento no existen mejoras significativas en el sistema auditivo, lo cual es atribuido al daño permanente que genera un estado crónico de esta enfermedad sin poder ser corregido con terapia farmacológica ${ }^{77}$. Los resultados de estos estudios se contraponen, parcialmente, a los resultados obtenidos por Di Girolamo y cols (2001) ${ }^{78}$ quienes evaluaron el efecto de la estimulación acústica contralateral (EAC) en pacientes con MG. En situación basal la EAC no induce cambios significativos en DPOAE, pero al ser evaluados 1 hora después de la administración oral de $60 \mathrm{mg}$ de piridostigmina se encontró una disminución significativa para algunas de las frecuencias evaluadas $^{78}$. Los autores demuestran así, que el aumento de disponibilidad de ACh dentro del espacio sináptico entre las fibras eferentes y las CCE permite restaurar la función de las $\mathrm{CCE}^{78}$. De acuerdo a estos antecedentes se puede concluir que la utilización de un inhibidor de acetilcolinesterasa reversible permite restituir la función eferente del sistema auditivo, siempre que el estadío de la enfermedad no involucre un daño permanente del oído. Concordante con esta visión, los resultados de Paludetti y cols $(2001)^{79}$ muestran que en pacientes con MG, la DPOAE y TOAE son significativamente menores comparado con el grupo control. Pero la utilización de piridostigmina incrementó significativamente la amplitud de estos registros para frecuencias medias y altas en pacientes con MG, aunque siendo significativamente menores en comparación a los del grupo control. Esta recuperación parcial (frecuencia especifica) posterior a la administración de piridostigmina podría estar explicada por la distribución de los receptores colinérgicos a nivel coclear ${ }^{77,79}$.

Los hallazgos demuestran que en pacientes con MG existe una disminución de la actividad eferente auditiva la cual puede ser parcialmente recuperada con la utilización de inhibidores reversibles de la colinesterasa ${ }^{77,79,80}$. Este efecto estaría dado por el aumento temporal de la disponibilidad de ACh, el principal neurotransmisor presente en las fibras eferentes que inervan las CCE. Este efecto es frecuencia dependiente, lo cual se relaciona con las áreas cocleares inervadas por las fibras del sistema eferente auditivo ${ }^{77,79}$. El efecto que puedan tener los inhibidores sobre el funcionamiento del eferente auditivo, tiene relación con la integridad del sistema auditivo y aparentemente está relacionado con el estado de avance de la enfermedad, la cual se asocia con cambios permanentes que no pueden ser tratados con terapia farmacológica ${ }^{77}$.

\section{(v) Déficit atencional}

El funcionamiento del sistema eferente auditivo también ha sido relacionado con desórdenes del com- 
portamiento tales como el déficit atencional (DA). De acuerdo al manual de diagnósticos y estadísticas de desórdenes mentales (DSM-IV) DA es definido como "un desorden del comportamiento que se origina en la infancia y cuyos síntomas son atención inapropiada, impulsividad e hiperactividad". Los niños que padecen DA presentan un patrón persistente de desatención e hiperactividad, el cual puede manifestarse en diferentes grados, impactando en el desarrollo social, laboral, ocupacional y académico $0^{81}$. La atención juega un rol en la selección de estímulos sensoriales, y en este sentido la atención permite diferenciar y entender el lenguaje en ambientes ruidosos ${ }^{81}$ ignorando el ruido de fondo a través de un mecanismo que involucra al sistema eferente $\mathrm{OCM}^{82}$. Esta observación ha permitido a algunos autores relacionar el DA con un inadecuado funcionamiento del sistema OCM. En este contexto, Pereira y cols (2012) ${ }^{81}$ evaluaron a través de la medición de TOAE la integridad del sistema eferente auditivo, sin encontrar diferencias entre pacientes con DA y el grupo control. Tampoco se encontró efecto en la supresión de la TOAE al introducir un ruido contralateral. De acuerdo a ello, los autores infieren que la pérdida de atención no es debida a defectos en el sistema eferente auditivo, aunque precisan que se requiere estudiar un mayor número de individuos ${ }^{81}$. Esta evidencia se complementa con estudios que han evaluado la función binaural en niños que padecen DA no encontrándose diferencias significativas con los grupos control83, a pesar que muestran una evidente disminución en la performance conductual que involucra atención auditiva. Si bien actualmente, no existe una fuerte evidencia que permita relacionar el DA con déficit de la percepción auditiva, la literatura científica lo ha relacionado íntimamente con desórdenes de procesamiento auditivo ${ }^{84}$.

De acuerdo a la posibilidad de estudiar el funcionamiento del sistema OCM de manera no invasiva y de la relativa importancia que se le ha otorgado al sistema auditivo eferente en la atención selectiva a estímulos auditivos, es que se ha relacionado disfunciones del eferente auditivo con el DA. Sin embargo, las evidencias hasta el momento no son lo suficientemente concluyentes para permitir establecer una relación.

\section{(vi) Mutismo selectivo}

El mutismo selectivo (MS) es una condición psicológica que usualmente afecta a niños, y cuya principal manifestación clínica es el silencio y no hablar en situaciones sociales específicas (en las que hablar es esperado), a pesar de que no exista la dificultad para realizarlo en otro contexto (DSMIV-TR). Descrito en el siglo XIX y originalmente denominado como "afasia voluntaria", se enfatiza en que la decisión de no hablar es voluntaria ${ }^{85}$. En este aspecto se ha ligado al MS con diversas comorbilidades que incluyen enuresis, encopresis, desorden obsesivo-compulsivo, depresión, retraso del desarrollo y síndrome de Asperger ${ }^{86}$. Sin embargo, resultados obtenidos los últimos años han permitido vincular el funcionamiento del sistema auditivo con esta patología. En animales de experimentación se ha encontrado evidencia de la existencia de circuitos inhibitorios en el sistema auditivo, que se activan en presencia de la propia voz, lo cual permite la preservación de la sensibilidad auditiva a estímulos externos ${ }^{87}$. Esta función, durante la vocalización, está operada por el reflejo acústico de oído medio (RAOM) y por actividad del eferente olivococlear ${ }^{88}$, permitiendo la desensibilización del sistema auditivo durante la vocalización. En este aspecto Bar-Haim y cols $(2004)^{89}$ proponen que en niños con MS podría existir un ineficiente funcionamiento del RAOM y una supresión eferente disminuida durante la vocalización, lo que resultaría en enmascaramiento de estímulos externos y desensibilización de la vía auditiva a sonidos externos, generando una negativa a hablar en ciertas situaciones sociales ${ }^{89}$. Las evidencias obtenidas del estudio de la función de RAOM y el funcionamiento del sistema eferente auditivo (estudiado a través de las TOAE) demostraron que estos pacientes tienen alteraciones en el funcionamiento del RAOM y una disminución de la función del sistema eferente auditivo, no existiendo diferencias en el funcionamiento de la vía auditiva aferente, lo cual fue constatado a través de las latencias de los potenciales de tronc $0^{89}$. Las evidencias encontradas por el grupo de Bar-Haim, permiten afirmar que en niños con MS el sistema eferente auditivo y la funcionalidad del RAOM, están deterioradas, generando dificultad en la escucha durante su propia vocalización. Esta condición asociada a ansiedad social, podría estar exacerbando una conducta de aislamiento en que el niño (en ocasiones) deja de comunicarse de forma hablada. Posteriormente, para poner a prueba esta posibili- 
dad un estudio evaluó si el procesamiento auditivo estaba deteriorado durante vocalizaciones en niños que sufrían de MS y que mostraban una actividad eferente auditiva anorma| ${ }^{88}$. En este estudio, fueron sometidos a pruebas de atención auditiva y visual 3 grupos de niños: control, un grupo de niños con diagnóstico de MS, pero con actividad eferente auditiva normal y un grupo diagnosticado con MS, pero con actividad eferente auditiva anormal. Los resultados mostraron que niños diagnosticados con MS y con eferente auditivo anormal obtuvieron un desempeño pobre, cometiendo más errores en las pruebas de atención auditiva (y no en la visual) en las cuales requerían vocalizar, respecto a niños con MS pero con actividad auditiva eferente normal ${ }^{88}$. Estos resultados demuestran que el sistema eferente auditivo cumple un importante rol en modular la percepción de la propia voz cuando simultáneamente se procesa información auditiva durante una conversación. Un sistema eferente auditivo anormal podría explicar, al menos en parte, la dificultad de niños con MS de poder comunicarse a través del lenguaje en ciertas situaciones sociales que involucran una mayor ansiedad. Estos descubrimientos generan una opción interesante de estudio con el fin de encontrar nuevos blancos farmacológicos para el tratamiento del MS.

\section{(vii) Otras patologías y sistema eferente auditivo}

Se ha postulado la presencia de disfunción olivococlear tipo neuropatía en sujetos que desarrollan diabetes mellitus. Específicamente, se han desarrollado modelos con ratas a las que se les induce diabetes y se compara la magnitud del reflejo eferente con ruido contralateral y se observa que 25 semanas después de la inducción de diabetes se reduce la magnitud del reflejo eferente en estas ratas diabéticas ${ }^{90}$.

Jacobs y cols (2012) $)^{91}$ evaluaron la amplitud del reflejo eferente auditivo durante la prueba de tolerancia a la glucosa en humanos, mostrando que el reflejo OCM inducido por ruido contralateral es afectado por los niveles de glicemia del paciente. De esta manera ellos demostraron la relación entre hiperglicemia, hiperinsulinismo y diabetes con la magnitud del reflejo eferente auditivo.

En relación a la migraña, Bolay y cols (2008) $)^{92}$ compararon la magnitud del reflejo olivococlear por ruido contralateral y encontraron que los pacientes con migraña presentan una reducción de la actividad eferente comparada a la de sujetos controles, a pesar de no existir diferencias en la audiometría y en la amplitud de los potenciales de tronco. De esta manera los autores postulan que la fonofobia presente en las crisis migrañosas podría deberse a una disfunción del sistema eferente auditivo.

\section{CONCLUSIONES}

En los últimos años se han descubierto nuevos neurotransmisores presentes en las sinapsis entre las fibras olivococleares mediales y las células ciliadas externas y entre las fibras olivococleares laterales y del nervio auditivo. Por otra parte, se han descrito las vías neuroanatómicas y algunos de los neurotransmisores de las vías descendentes que provienen de la corteza auditiva y modulan la actividad del sistema olivococlear. Probablemente en un futuro cercano, podríamos estar en presencia del desarrollo de nuevos tratamientos farmacológicos que modulen las vías eferentes y receptores en forma específica inhibiendo, activando o modulando el sistema eferente auditivo permitiendo así mejorar las diversas patologías producidas por una disfunción eferente.

\section{Agradecimientos}

Parte del trabajo presentado en este artículo fue financiado por fondos concursables de investigación de la Sociedad Chilena de Otorrinolaringología y por el proyecto Fondecyt 1120256.

\section{BIBLIOGRAFÍA}

1. Rasmussen GL. The olivary peduncle and other fiber projections of the superior olivary complex. J Comp Neurol 1946; 84: 141-219.

2. RASMUSSEN GL. Efferent fibers of the cochlear nerve and cochlear nucleus. En: Rasmussen GL, Windle WF. eds. Neural mechanisms of the auditory and vestibular systems. Springfield, IL: Thomas; 1960; 105-15.

3. WarR WB, Guinan JJ JR. Efferent innervation of the organ of Corti: Two separate systems. Brain Res 1979; 173: 152-5. 
4. Délano P, Robles I, Robles L. Sistema eferente auditivo. Rev Otorrinolaringol 2005; 55-62.

5. Guinan JJ. JR. Olivocochlear Efferents: Anatomy, Physiology, Function, and the Measurement of Efferent Effects in Humans. Ear Hear 2006; 27(6): 589-607.

6. Robles L. and Delano PH. Efferent System, in "The Senses: a Comprehensive Reference", Chapter 13, Volume: Audition, Editors P. Dallos, and D. Oertel. Elsevier Press, 2008; pags 413-45.

7. Schofield BR. Central descending auditory pathways. Auditory and vestibular efferents. New York: Springer. 2010; pp 261-90.

8. Maison SF, Liberman MC. Predicting vulnerability to acoustic injury with a noninvasive assay of olivocochlear reflex strength. J Neurosci 2000; 20(12): 4701-7.

9. Kawase T, Liberman MC. Antimasking effects of the olivocochlear reflex. I. Enhancement of compound action potentials to masked tones. J Neurophysiol 1993; 70: 2519-32.

10. OAtman LC. Role of visual attention on auditory evoked potentials in unanesthetized cats. ExpNeurol 1971; 32: 341-56.

11. Délano PH, Elgueda D, Hamame CM, Robles L. Selective attention to visual stimuli reduces cochlear sensitivity in chinchillas. J Neurosci2007; 27: 4146-53.

12. Velluti R, Pedemonte M, García-Austt E. Correlative changes of auditory nerve and microphonic potentials throughout sleep. Hear Res 1989; 39: 203-8.

13. Robles L, Delano PH. Efferent System. The Senses: A Comprehensive Reference. London: Academic Press 2008; 413-445 p.

14. Ryugo DK. Introduction to efferent Systems, in "Auditory and Vestibular Efferents", Chapter 1, Volume 38, Editors: Ryugo DK, Fay RR, Popper AN Springer HandBook of auditory Research. 2011; pags. 1-12.

15. WARR WB. Olivocochlear and vestibular efferent neurons of the feline brain stem: their location, morphology and number determined by retrograde axonal transport and acetylcholinesterasehistochemistry. J Comp Neurol 1975; 161: 159-82.

16. Puel JL. Chemical synaptic transmission in the cochlea. Progress in Neurobiology 1995; 47(6): 449-76.
17. Eybalin M, Carachon G, Renard N. Dopaminergic lateral efferent innervation of the guineapig cochlea: immunoelectron microscopy of catecholamine-synthesizing enzymes and effect of 6-hydroxydopamine. Neuroscience 1993; 54(1): 133-42.

18. Eybalin M. Neurotransmitters and neuromodulators of the mammalian cochlea. Physiol Rev 1993; 73(2): 309-73.

19. Saffiedine S, Soto Prior AM, Eybalin M. Choline Acetyltransferase, Glutamate Decarboxylase. Tyrosine Hydroxylase, Calcitonin Gene-related Peptide and Opioid Peptides Coexist in Lateral Efferent Neurons of Rat-and Guinea-pig. Eur J Neurosci 1997; 9(2): 356-67.

20. Vetter DE, Katz E, Maison SF, Taranda J, Turcan S, BALLESTERO $\mathrm{J} Y$ COLS. The $\alpha 10$ nicotinic acetylcholine receptor subunit is required for normal synaptic function and integrity of the olivocochlear system. Proc Natl Acad Sci 2007; 104(51): 20594-9.

21. Altschuler Ra, Kachar B, Rubio Ja, Parakkal $\mathrm{MH}$, FEz J. Immunocyto chemical localization of choline acetyltransferase-like immunoreactivity in the guinea pig cochlea. Brain Res 1985; 338(1): 1-11.

22. Eybalin M, Pujol R. Choline acetyltransferase (ChAT) immunoelectron microscopy distinguishes at least three types of efferent synapses in the organ of Corti. Exp Brain Res 1987; 65(2): 261-70.

23. Raphael Y, Altschuler RA. Structure and innervation of the cochlea. Brain Res Bull 2003; 60(5-6): 397-422.

24. Rogers SW, Myers JE, Gahring LC. The expression of nicotinic receptor alpha 7 during cochlear development. Brain and Behavior 2012; 2(5): 62839.

25. Gil-Loyzaga P, Parés-Herbute N. HPLC detection of dopamine and noradrenaline in the cochlea of adult and developing rats. Brain Res Dev Brain Res 1989; 48(1): 157-60.

26. D'aldin C, Puel Jl, Leduco R, Crambes 0, Eybalin M, PujoL R. Effects of a dopaminergic agonist in the guinea pig cochlea. Hear Res 1995; 90(1-2): 20211.

27. D'aldin C, Eyebalin M, Puel Jl, Charachon G, Ladrech S, Renard N, Pujol R. Synaptic connections and putative functions of the dopaminergic innervation of the guinea pig cochlea. Eur Arch Otorhinolayngol 1995; 252(5): 270-4. 
28. Ruel J, Nouvian R, GervaisD'aldin C, Pujol R, Eybalin M, PuEL JL. Dopamine inhibition of auditory nerve activity in the adult mammalian cochlea. Eur $\mathrm{J}$ Neurosci 2001; 14(6): 977-86.

29. FEXJ, Altschuler RA. Enkephalin-like immunoreactivity of olivocochlear nerve fibers in cochlea of guinea pig and cat. Proc Natl Acad Sci 1981; 78(2): 1255-9.

30. Hoffman DW, Zamir N, Rubio J, Altschuler RA, FEX J. Proenkephalin and prodynorphin related neuropeptides in the cochlea. Hear Res 1985; 17: 47-50

31. Katauli M, Yamashita T, Tohyama Y, Kumazawa T, Takeda $\mathrm{N}$, NAWASAKI Y Y coLs. Localization of calcitonin generelated peptide in the organ of Corti of the rat: an immunohistochemical study. Brain Res 1985; 358(1-2): 394-7.

32. Matsunaga T, Dol K, Takeda N, Mori N and Tohyama M. Effect of calcitonin gene-related peptide (CGRP) on compound action potential of the auditory nerve of guinea pigs (abstr.). Colhgium Oto-RhinoLaryngologicum Amicitiae Sacrum, Hakone, Japan, 1986; p. 30.

33. Adams JC, Mugniani E. Patterns of glutamate decarboxylase immunostaining in the feline cochlear nuclear complex studied with silver enhancement and electron microscopy. J Comp Neurol 1987; 262(3): 375-401.

34. Adams JC, Mroz EA, Sewell WF. A possible neurotransmitter role for CGRP in a hair-cell sensory organ. Brain Res 1987; 419(1-2): 347-51.

35. Thompson AM, Thompson GC. Light microscopic evidence of serotoninergic projections to olivocochlear neurons in the bush baby (Otolemurgarnettii). Brain Res 1995; 695: 263-6.

36. Gil-Loyzaga P, Bartolomé MV, Vicente-Torres MA. Serotonergic innervation of the organ of Corti of the cat cochlea. Neuroreport 1997; 8(16): 3519-22.

37. Vicente-Torres A, Bartolomé MV, Carricondo F, Esquifino A, Gil-Loyzaga P. HPLC detection of serotonin within the rat cochlea. Neuroreport 1998; 9(16): 3699-701.

38. Gil-Loyzaga P, Bartolomé V, Vicente-Torres A, Carricondo F. Serotonergic innervation of the organ of Corti. Acta Otolaryngol 2000; 120(2): 128-32.

39. Bartolomé MV, Gil-Loyzaga P. Serotonergic innervation of the inner ear: is it involved in the general physiological control of the auditory receptor? Int Tinnitus J 2005; 11(2): 119-25.

40. Dlugaiczyk J, Singer W, Schick B, Iro H, Becker K,
BECKER CM. Expression of glycine receptors and gephyrin in the rat cochlea. Histochem Cell Biol 2008; 129: 513-23.

41. Buerbank S, Becker K, Becker CM, Brandt N, Engel J, KNIPPER M. Developmental regulation of glycine receptors at efferent synapses of the murine cochlea. Histochem Cell Biol 2011; 136: 387-98

42. GUINAn JJ JR, Stankovich KM. Medial efferent inhibition produces the largest equivalent attenuations at moderate to high sound levels in cat auditory-nerve fibers. J Acoust Soc Am 1996; 100(3): 1680-90.

43. SpoendLIN H. [Noise and cochlea]. JFORL J Fr Otorhinolaryngol Audiophonol Chir Maxillofac 1972; 21(2): 107-13.

44. SpoendLIN H. Innervation densities of the cochlea. Acta Otolaryngol 1972; 73(2): 235-48.

45. Elgoyhen AB, Johnson DS, Boulter J, et al. Alpha 9: an acetylcholine receptor with novel pharmacological properties expressed in rat cochlear hair cells. Cell 1994; 79: 705-15.

46. Elgoyhen AB, KatzE, Fuchs PA. The nicotinic receptor of cochlear hair cells: a possible pharmacotherapeutic target? Biochem Pharmacol 2009; 78(7): 712-9.

47. Dulon D, Luo L, Zhang C, Ryan AF. Expression of small-conductance calcium-activated potassium channels (SK) in outer hair cells of the rat cochlea. Eur J Neurosci 1998; 10(3): 907-15.

48. Oliver D, Kloker N, Schuck J, Baukrowitz T, Ruppersber G, Fakler B. Gating of Ca21-Activated $\mathrm{K} 1$ channels controls fast inhibitory synaptic transmission at auditory outer hair cells. Neuron 2000; 26: 595-601.

49. Elgoyhen AB, Katz E. The efferent medial olivocochlear-hair cell synapse. J Physiol Paris 2012; 106(1-2): 47-56.

50. GitTer AH, Zenner HP. $\gamma$-Aminobutyric acid receptor activation of outer hair cells in the guinea pig cochlea. Eur Arch Otorhinolaryngol 1992; 249: 62-5.

51. KuriYAma K, HiRouchi M. Structure and function of GABA receptor in the brain. Tanpakushitsu Kakusan Koso 1990; 35(8): 1455-64.

52. Maison Sf, Emeson RB, Adams JC, Luebke AE, LiBerman MC. Loss of alpha CGRP reduces sound-evoked activity in the cochlear nerve. $J$ Neurophysiol 2003; 90(5): 2941-9.

53. Feliciano M, Saldaña E, Mugnaini E. Direct projections from rat primary auditory neocortex to nucleus sagulum, paralemniscal regions, 
superior olivary complex and cochlear nuclei. Aud Neurosci 1995; 1: 287-308.

54. Doucet JR, Rose L, Ryugo DK. The cellular origin of corticofugal projections to the superior olivary complex in the rat. Brain Res 2002; 925: 28-41.

55. MuLders WH, RoberTson D. Evidence for directcortical innervation of medial olivocochlearneurones in rats. Hear Res 2000; 144(1-2): 65-72.

56. Saldaña E, Feliciano M, Mugnaini E. Distribution of descending projections from primary auditory neocortex to inferior colliculus mimics the topography of intracollicular projections. J Comp Neurol 1996; 371(1): 15-40.

57. Schofield BR. Central descending auditory pathways. Auditory and vestibular efferents. New York: Springer. 2010; pp 261-90.

58. Heft। BJ, Sмiтh PH. Anatomy, physiology, and synaptic responses of rat layer $V$ auditory cortical cells and effects of intracellular GABA(A) blockade. J Neurophysiol 2000; 83(5): 262638.

59. Thompson AM, Thompson GC. Relationship of descending inferior colliculus projections to olivocochlear neurons. J Comp Neurol 1993; 335(3): 402-12.

60. Feliciano M, Potashner SJ. Evidence for a glutamatergic pathway from the guinea pig auditory cortex to the inferior colliculus. $J$ Neurochem 1995; 65(3): 1348-57.

61. XIAO Z, Suga N. Modulation of cochlear hair cells by the auditory cortex in the mustached bat. Nat Neurosci 2002; 5(1): 57-63.

62. León A, Elgueda D, Silva Ma, Hamamé CM, Delano $\mathrm{PH}$. Auditory cortex basal activity modulates cochlear responses in chinchillas. PLoSOne 2012; 7(4): 1-13.

63. Perrot $X$, Ryvlin P, Isnard J, Guénot M, Catenoix H, Fischer C, Mauguière F, Collet L. Evidence for corticofugal modulation of peripheral auditory activity in humans. Cereb Cortex 2006; 16(7): 941-8.

64. Riga M, Papadas T, Werner Ja, Dalchow CV. A Clinical Study of the Efferent Auditory System in Patients With Normal Hearing Who Have Acute Tinnitus. Otol Neurotol 2007; 28: 185-90.

65. Geven LI, de KleineE, Free RH, van DiJk P. Contralateral suppression of otoacoustic emissions in tinnitus patients. Otol Neurotol 2011; 32: 315-21.

66. Paglialonga A, Fiocchi S, Del Bo L, Ravazzani P,
TOGNOLA G. Quantitative analysis of cochlear active mechanisms in tinnitus subjects with normal hearing sensitivity: Time-frequency analysis of transient evoked otoacoustic emissions and contralateral suppression. Auris Nasus Larynx 2011; 38: 33-40.

67. Geven LI, Wit HP, de Kleine E, van Dijk P. Wavelet analysis demonstrates no abnormality in contralateral suppression of otoacoustic emissions in tinnitus patients. Hear Res 2012; 286: $30-40$.

68. Suckfüll M, Althaus M, Ellers-Lenz B, Gebauer A, Görtelmeyer R, Jastreboff PJ, Moebius hJ, Rosenberg T, Russ H, Wirth Y, Krueger H. A randomized, double-blind, placebo-controlled clinical trial to evaluate the efficacy and safety of neramexane in patients with moderate to severe subjective tinnitus. BMC Ear Nose Throat Disord 2011; $11: 1$.

69. Fetoni AR, Picciotti PM, Paludetti G, Trolani D. Pathogenesis of presbycusis in animal models: a review. Exp Gerontol 2011; 46: 413-25.

70. Stamataki S, Francis HW, Lehar M, May BJ, Ryugo DK. Synaptic alterations at inner hair cells precede spiral ganglion cell loss in aging C57BL/6J mice. Hear Res 2006; 221: 104-18.

71. Fu B, Le Prell C, Simmons D, Lei D, Schrader A, CHen $A B, B$ AO J. Age-related synaptic loss of the medial olivocochlear efferent innervation. Molecular Neurodegeneration 2010; 5: 53.

72. Jacobson M, Kim S, Romney J, Zhu X, Frisina $\mathrm{RD}$. Contralateral suppression of distortionproduct otoacoustic emissions declines with age: a comparison of findings in CBA mice with human listeners. Laryngoscope 2003; 113: 1707-13.

73. Zhu X, VasilyeVa ON, KIm S, Jacobson M, Romney J, Waterman MS, Tuttle D, Frisina RD. Auditory efferent feedback system deficits precede age- related hearing Ioss: contralateral suppression of otoacoustic emissions in mice. J Comp Neurol 2007; 503(5): 593-604.

74. Park SN, Back SA, Choung YH, Kim HL, AkiL O, Lustig LR, Park KH, YeO SW. $\alpha$-Synuclein deficiency and efferent nerve degeneration in the mouse cochlea: a possible cause of early-onset presbycusis. Neurosci Res 2011; 71: 303-10.

75. Lalaki P, Hatzopoulos S, Lorito G, Kochanek K, Sliwa $L$, SkarZYNSKI H. A connection between the Efferent Auditory System and Noise-Induced Tinnitus 
Generation. Reduced contralateral suppression of TEOAEs in patients with noise-induced tinnitus. Med Sci Monit 2011; 17(7): MT56-62.

76. Taranda J, Maison SF, Ballestero Ja, Katz E, Savino J, Vetter DE, Boulter J, Liberman MC, Fuchs PA, ELGOYHEN AB. A point mutation in the hair cell nicotinic cholinergic receptor prolongs cochlear inhibition and enhances noise protection. PLOS Biol 2009; 7(1): e18.

77. Hamed SA, Elattar AM, Hamed EA. Irreversible cochlear damage in myasthenia gravis - otoacoustic emission analysis. Acta Neurol Scand 2006; 113: 46-54.

78. Di Girolamo S, d'Ecclesia A, Quaranta N, Garozzo A, Evoli A, Paludetti G. Effects of contralateral white noise stimulation on distortion product otoacoustic emissions in myasthenic patients. Hear Res 2001; 162: 80-4.

79. Paludetti G, Di Nardo W, d’Ecclesia A, Evoli A, Scarano E, Di Girolamo $S$. The Role of Cholinergic Transmission in Outer Hair Cell Functioning Evaluated by Distortion Product Otoacoustic Emissions in Myasthenic Patients. Acta Otolaryngol 2001; 121: 119-21.

80. Toth L, Racz T, Dioszeghy P, Repassy G, Lampe I. Otoacoustic emission in myasthenia gravis patients and the role of efferent activation. Hear Res 1998; 126 : 123-5.

81. Pereira VR, Feitosa MÂ, Pereira LH, Azevedo MF. Role of the medial olivocochlear system among children with ADHD. Braz J Otorhinolaryngol 2012; 78(3): 27-31.

82. Kauramäkı J, JäÄskeläInen IP, Hänninen JL, Auranen T, Nummenmaa A, Lampinen J, Sams M. Two-stage processing of sounds explains behavioral performance variations due to changes in stimulus contrast and selective attention: an MEG study. PLOS One 2012; 7(10): e46872.
83. Pillsbury hC, Grose JH, Coleman WL, Conners CK, HaLL JW. Binaural function in children with attentiondeficit hyperactivity disorder. Arch Otolaryngol Head Neck Surg 1995; 121(12): 1345-50.

84. Freyaldenhoven MC, Thelin JW, Plyler PN, Nabelek AK, BURCHFIELD SB. Effect of stimulant medication on the acceptance of background noise in individuals with attention deficit/hyperactivity disorder. J Am Acad Audiol 2005; 16(9): 677-86.

85. KrYsanskI VL. A brief review of selective mutism literature. J Psychol 2003; 137(1): 29-40.

86. Wong P. Selective mutism: a review of etiology, comorbidities, and treatment. Psychiatry (Edgmont) 2010; 7(3): 23-31.

87. Hor R. Neurobiology: tuning in by turning off. Nature 2002; 418: 831-2.

88. Arie M, Henkin Y, Lamy D, Tetin-Schneider S, Apter A, SADEH A, Bar-HaIm Y. Reduced auditory processing capacity during vocalization in children with Selective Mutism. Biol Psychiatry 2007; 61(3): 419-21.

89. Bar-Haim Y, Henkin Y, Ari-Even-Roth D, Tetin-Schneider S, Hildesheimer M, Muchnik C. Reduced Auditory Efferent Activity in Childhood Selective Mutism. Biol Psychiatry 2004; 55: 1061-8.

90. Wu HP, Guo YL, Cheng TJ, Hsu CJ. Chronological changes in compromised olivocochlear activity and the effect of insulin in diabetic Wistar rats. Hear Res 2010; 270(1-2): 173-8.

91. Jacobs PG, Konrad-Martin D, McMillan GP, McDermott D, Fausti SA, Kagen D, Wan EA. Influence of acute hyperglycemia on otoacoustic emissions and the medial olivocochlear reflex. J Acoust Soc Am 2012; 131(2): 1296-306.

92. Bolay H, Bayazit YA, Gündüz B, Ugur AK, AkÇal D, Altunyay S, ILICA S, BabACAn A. Subclinical dysfunction of cochlea and cochlear efferents in migraine: an otoacoustic emission study. Cephalalgia 2008; 28(4): 309-17. 